\title{
NEUROSCIENCE
}

\section{Speeding up screening in smaller zebrafish}

Oprișoreanu, A. et al. Dis Model Mech 14, dmm047761 (2021)

Synapses are a critical part of neurons. In many motor neuron diseases, these junctions are often affected early on; as they become destabilized, neurodegeneration soon follows. Drugs that can stabilize synapses could potentially help patients but first, appropriate candidates need to be identified.

This is slow and costly to do in mouse models, so many researchers turn to optically transparent larval zebrafish. These can yield quicker insights into whether a compound is providing any benefit, but it still takes a time to screen chemical libraries in zebrafish. Ana-Maria Oprișoreanu and her colleagues at the University of Edinburgh have been working to speed that up.

Her colleagues had previously developed an automated system for drug screening in zebrafish, in which dosed fish are picked up by a robotic sampler and pushed through a tube under a spinning disk confocal microscope, which rotates and images them for analysis by a VAST BioImager.
The platform had however been developed for older, hardier, and more pigmented fish - about 48 hours post fertilization (hpf).

Oprişoreanu, who had been studying synapse plasticity in mice, wanted to look at younger fish in which the chondrolectin gene (chodl) had been knocked out. In mouse models of spinal muscular atrophy, this is one of the first genes to become dysregulated and in zebrafish, it is necessary for synapses to mature; immature synapses yield truncated axons. This phenotype, however, is most obvious at about $28 \mathrm{hpf}$, when the fish are very small, very fragile, and have very little pigmentation - a point of confusion for the computer. "The system was kind of like, what is that?" says Oprişoreanu. "Is it an air bubble, or a fish?"

With some tweaks to pumping parameters and object detection settings, Oprişoreanu and her colleagues calibrated the system to automatically process $28 \mathrm{hpf}$ zebrafish. That cut the screening time for 20 compounds in 144 embryos in half to just four hours. A screen of 982 compounds yielded four that helped rescue axon length. The most effective was dipyridamole, which also helped rescue the ubiquitin-like modifier activating enzyme I (UBA1) zebrafish model of SMA, which have a wider range of axonal abnormalities than chodl knockouts.

The platform takes some special equipment and parameters may vary depending on the mutant, but Oprişoreanu hopes the ability to quickly screen younger fish will help labs narrow in on potentially beneficial drugs that can then be taken on to mouse models and beyond. She, however, plans to stick with the fish. "It was very easy to switch," she says, noting the benefits for studying neurons that come with the simplicity and transparency of zebrafish. "You can immediately see if the axons are there!"

\section{Ellen P. Neff}

Published online: 21 June 2021

https://doi.org/10.1038/s41684-021-00800-4

\section{nature research} \section{EDITING SERVICE}



Feel confident writing in English with Nature Research language editing

$\Rightarrow$ Learn more at authorservices.springernature.com/language-editing 